\title{
Following a Low Carbohydrate, High Fat Diet Compared to Reduced Calorie, High Carbohydrate Diet as a Nutritional Intervention in Type Two Diabetes Mellitus Patients: A Systematic Review
}

\author{
Joy Lewis MS, RD, LD (Corresponding author) \\ Health and Human Performance Department, University of Houston, Houston, Texas \\ E-mail: jdlewis8@crimson.ua.edu
}

Kevin Haubrick PhD, RD, LD, FAND

Health and Human Performance Department, University of Houston, Houston, Texas

E-mail: khaubrick@uh.edu

Received: April 14, 2020 Accepted: May 14, 2020 Published: May 16, 2020

doi:10.5296/jfs.v9i1.17045 URL: https://doi.org/10.5296/jfs.v9i1.17045

\begin{abstract}
There is evidence supporting individuals with type 2 diabetes benefit from lifestyle changes through a nutrition intervention that improves diabetic (blood glucose and HgbAlc) and cardiovascular (total cholesterol, HDL, LDL, and triglycerides) biomarkers. The objective of this systematic review was to evaluate if patients with type 2 diabetes following a low carbohydrate, high fat eating pattern is more effective than following a reduced caloric, high carbohydrate eating pattern in the improvement of diabetic (blood glucose and HgbA1c) and cardiovascular (total cholesterol, HDL, LDL, and triglycerides) biomarkers. A literature search was conducted on peer-reviewed research trials registered in PubMed, from January 2007 to September 2019 using combinations of the search terms: Diabetes Mellitus, Type 2 AND Diet, Ketogenic; OR Diet, Carbohydrate-Restricted. The literature was analyzed in chronological order; grouping in four year increments from 2007 to 2019. The thirty-six articles reviewed provide evidence to support the use of a low carbohydrate diet in patients with type 2 diabetes versus a reduced caloric diet. This systematic review highlighted diabetic (HgbAlc and fasting blood glucose) and cardiovascular biomarkers (HDL) of type 2 diabetic
\end{abstract}


patients improve significantly when following a low-carbohydrate, high fat diet versus a reduced calorie, high carbohydrate intake.

Keywords: Low-carbohydrate, Type 2 diabetes mellitus, Dietary intervention, Calorie-restricted, American Diabetes Association

\section{Introduction}

Type 2 diabetes mellitus has many recommended treatment options including weight loss, carbohydrate counting, medications, or sliding scale insulin. A lifestyle intervention that includes physical activity, pharmacology, and nutrition interventions can lead to improved diabetic and cardiovascular biometrics (American Diabetes Association, 2015; Bhanpuri et al., 2018; Saslow, Daubenmier et al., 2017). Presently, there is not a specific diet to provide as a nutritional intervention for patients with type 2 diabetes. A person with diabetes should receive recommendations that take into account their medical history, personal preferences, and metabolic goals (American Diabetes Association, 2020). Generally, it is recommended to eat balanced meals that include fruits and vegetables, high fiber, lean proteins, and whole grains. The goal is to not only focus on reducing carbohydrates, but eating less processed carbohydrates, and added sugars (American Diabetes Association, 2020).

Recommendations also differ in what will result in weight loss, glycemic control, and improved cardiovascular profile (Elhayany, Lustman, Abel, Attal-Singer, \& Vinker, 2010). The American Diabetic Association (ADA) recommends health care providers individualize the diet to fit the needs of the patient (American Diabetes Association, 2015; American Diabetes Association, 2020). When developing nutrition intervention(s) it is important to factor in the possible benefits or detractors to the patient. This literature review will evaluate if patients with type 2 diabetes following a low carbohydrate, high fat eating pattern is more effective than following a reduced caloric, high carbohydrate intake eating pattern in the improvement of diabetic (blood glucose and HgbA1c) and cardiovascular biomarkers (total cholesterol, high density lipoproteins [HDL], low density lipoproteins [LDL], and triglycerides). The literature will be analyzed in chronological order; grouping in four year increments from 2007 to 2019. From this point forward the use of the word diabetes will be referring to type 2 diabetes, unless otherwise specified.

\section{Pathophysiology of Diabetes}

Type 2 diabetes is the insulin resistant form usually caused by overproduction of insulin due to hyperglycemia (Francois et al., 2018; Hou et al., 2018; Myette-Cote et al., 2018; Wang et al., 2018). Hyperglycemia subsequently caused by obesity, sedentary lifestyle, unhealthy eating habits, or other metabolic conditions. People who have diabetes usually present with dyslipidemia, hypertension and hyperinsulinemia (Fernemark et al., 2013; Sanada et al., 2018; Tay et al., 2014). Traditionally diabetes is treated with a low fat, reduced caloric intake diet that promotes weight loss (Dyson, Beatty, \& Matthews, 2007; Esposito et al., 2009; Guldbrand et al., 2012; Haimoto, Iwata, Wakai, \& Umegaki, 2008; McLaughlin et al., 2007). A 5-10\% weight loss has shown to improve glycemic control and reduce heart disease (American Diabetes Association, 2015). A low carbohydrate diet is thought to help patients with diabetes because the reduced carbohydrate intake causes insulin to not be overproduced. 


\section{MInstitute ${ }^{\text {Macrothink }}$}

The reduced insulin production allows the cells to uptake the glucose in the blood and lower the blood sugar. Additionally, a diet high in protein and fat can increase satiety and does not prompt the release of insulin (Bhanpuri et al., 2018; Dashti et al., 2007; Hamdy et al., 2018; Samkani et al., 2018).

\section{Literature Search Strategy}

PubMed was searched using the dates of January 2007 to September of 2019 using combinations of the search terms: Diabetes Mellitus, Type 2 AND Diet, Ketogenic; OR Diet, Carbohydrate-Restricted. Filters were added to show only studies in English, with humans, and peer-reviewed journal articles. The search resulted in 186 articles. One investigator independently screened the articles and performed the data extraction. Articles were screened and excluded if not original research, did not include participants diagnosed with type 2 diabetes, and did not use a low carbohydrate diet or ketogenic diet as an intervention. All trials completed on animals, children, or pregnant women were excluded. Thirty-six peer reviewed trials were included. The Preferred Reporting Items for Systematic review and Meta-Analysis Protocols (PRISMA-P) 2015 checklist (Shamseer et al., 2015) was used to complete the systematic review.

\section{2007 to 2010}

From 2007 to 2010, research on a low carbohydrate dietary intervention in type 2 diabetes was in the infancy stage. The 2007 Standards of Medical Care for Diabetes, authored by the American Diabetes Association, had diagnoses and recommendations that have significant variation from the current 2020 Standards. The most notable variation is the use of the HgbA1c. According to the 2007 American Diabetes Association Standards, a patient was diagnosed with diabetes if one of the following was present: symptoms of diabetes with casual plasma glucose (CPG) greater than or equal to $200 \mathrm{mg} / \mathrm{dl}$, fasting plasma glucose (FPG) greater than or equal to $126 \mathrm{mg} / \mathrm{dl}$, or greater than or equal $200 \mathrm{mg} / \mathrm{dl}$ after two hours during the oral glucose tolerance test (American Diabetes Association, 2007). The HgbA1c was considered unreliable and only used as a tool to monitor glucose levels after diagnosis (American Diabetes Association, 2007).

Lifestyle modification was recommended to be individualized to each patient, but there was a standard diet recommendation. Medical nutrition therapy for patients with diabetes was a diet promoting weight loss in overweight/obese patients with the focus on fat intake less than $30 \%$ of calories for the day (American Diabetes Association, 2007). Carbohydrate counting and monitoring was recommended. Carbohydrate counting is calculating the total number of grams of carbohydrates needed per day. Then through education, the patient is able to allocate grams of carbohydrates per meal and snacks to help manage their blood sugar. Carbohydrate intake was recommended to be $50 \%$ of overall calories (American Diabetes Association, 2007). A low carbohydrate diet is not recommended as a treatment option due to the lack of long term research supporting this recommendation (American Diabetes Association, 2007). At the time the short term results of the research highlighted weight loss, but inconclusive results of improvement in cardiac biomarkers (American Diabetes Association, 2007; Dashti et al., 2007; McLaughlin et al., 2007). 


\subsection{Clinical Trials}

To the knowledge of the researchers, Mclaughlin (2007) was reported as the first trial completed utilizing a low carbohydrate and high fat diet for research. This is worth noting because the study directly contradicts the ADA recommendations at the time (American Diabetes Association, 2007; McLaughlin et al., 2007). Conversely, while the team embarked on new territory their study design was not followed by other researchers. The study included 29 participants who were placed on a hypocaloric diet. The participants were in a 750 calorie deficit. This trial lasted 16 weeks and resulted in 7\% weight loss amongst participants and significant improvements in plasma glucose, insulin, and triglyceride concentrations. Table 1 shows the nutrition interventions used from 2007 to 2010. The table notes low carbohydrate diet definition varies by research team, and limits the reproducibility of these studies in the future. During this time period, research referenced the ADA guidelines as the basis for the control diet in their study (Elhayany et al., 2010; Haimoto et al., 2008; Iqbal et al., 2010). The control diet consisted of at least $50 \%$ of calories from carbohydrates, $30 \%$ from fat, and a caloric deficit to promote weight loss. Comparing this to a low carbohydrate diet is more representative of the nutritive intervention provided to patients during the time.

Table 1. Description of low carbohydrate interventions in studies of participants with type 2 diabetes 2007 to 2010

Description of low carbohydrate interventions in studies of participants with type 2 diabetes 2007 to 2010

\section{Reference Population}

Dashti et al. 64 obese participants with (2007) Type 2 diabetes and participants with normal blood glucose levels

McLaughlin et al. (2007)

29 overweight or obese participants with fasting plasma glucose concentration $\quad 7.2-8.3$ $\mathrm{mmol} / \mathrm{l}$, taking no antihyperglycemic medications, and stable body weight for the 3 months prior to the trial

\section{Study Authors' description of nutrition Length intervention}

56 weeks

16 weeks

Hypocaloric low carbohydrate diet defined as a 750 calorie deficit daily, coupled with $40 \%, 15 \%$, and $45 \%$ of calories from $\mathrm{CHO}$, protein, and fat respectively 
al. (2008)

diagnosed with type 2 diabetes being seen in an outpatient clinic

De Natale 18 overweight/obese et al. (2009) participants with type 2 diabetes, with satisfactory blood glucose control through diet or metformin and diet

Dyson et al. 26 (2009)

overweight/obese 3 participants with a group of 13 with type 2 diabetes (on a diet or metformin) and 13 without type 2 diabetes

Esposito et 215 overweight al. (2009) participants with type 2 diabetes, never treated with antihyperglycemic drugs, sedentary, and had hemoglobin $\mathrm{A}(1 \mathrm{c})$ levels less than $11 \%$

Elhayany et $179 \quad$ (93 men and 86 al. (2010) women) overweight/obese participants with type 2 diabetes patients, $\mathrm{HbAlc}$ measurement $7-10 \%$, no change in diabetes medication for at least 3 months

Iqbal et al. 144 obese participants with (2010) type 2 diabetes, military veterans, and using insulin or antidiabetic medications
$33 \%$ of calories from carbohydrates, protein, and fat respectively

4 Low $\mathrm{CHO} /$ high MUFA diet defined as weeks $45 \%$ of calories from carbohydrates and $23 \%$ of calories from MUFA

Low CHO diet defined as less than or equal to $40 \mathrm{~g}$ of $\mathrm{CHO}$ a day

4 years Mediterranean-style low $\mathrm{CHO}$ diet defined as less than $50 \%$ of daily calories from $\mathrm{CHO}$

12 months

Low CHO Mediterranean diet defined by a macronutrient composition of $35 \%$ $\mathrm{CHO}, 45 \%$ fat, and $20 \%$ protein of calories eaten daily
24

months
Low CHO diet defined as less than 30 grams of $\mathrm{CHO}$ per day

Dashti et al. (2007), Dyson et al. (2007), and Iqbal et al. (2010) had the most restrictive interventions, which reduced the carbohydrate intake to less than 40 grams per day. The remaining studies listed in Table 1 reduced the calories from carbohydrates from $50 \%$ to a range of $35 \%$ to $45 \%$ (De Natale et al., 2009; Elhayany et al., 2010; Esposito et al., 2009; 


\section{$\triangle$ Macrothink}

Haimoto et al., 2008; McLaughlin et al., 2007). The variation in the amount of carbohydrates in the diet showed a small reduction in carbohydrates may be just as beneficial as an extremely low carbohydrate diet. Researchers used Registered Dietitians (RD) to complete dietary recalls on patients (De Natale et al., 2009; Dyson et al., 2007; Elhayany et al., 2010; Esposito et al., 2009; Haimoto et al., 2008), while other studies relied on self-reported recall (Dashti et al., 2007; Dyson et al., 2007; Elhayany et al., 2010; Haimoto et al., 2008; Iqbal et al., 2010; McLaughlin et al., 2007). All study designs during this time period did not control for exercise. This is important to point out because exercise of at least 150 minutes per week can reduce risk for cardiovascular disease, and promote weight loss.

\subsection{Results}

The effect of a reduced carbohydrate diet intervention shows potential improvements in cardiovascular and diabetic biomarkers in patients. All studies with HgbAlc as an outcome variable noted a statistically significant reduction $(p<0.05)$, when compared to the control group or low fat diet (Dashti et al., 2007; Dyson et al., 2007; Elhayany et al., 2010; Esposito et al., 2009; Haimoto et al., 2008). This is important to note because HgbAlc generally represents the last two to three months (Mahan \& Raymond, 2016). The trials by Esposito et al (2009) and Haimoto et al (2008) lasted for two and four years respectively. The significant reduction shows sustained blood glucose control over a prolonged period of time, and patients are able to remain compliant.

Cardiovascular biomarkers improved significantly $(p<0.05)$ in the low carbohydrate versus the control group (De Natale et al., 2009; Elhayany et al., 2010; McLaughlin et al., 2007). There was an statistically significant $(\mathrm{p}<0.05)$ increase of the high-density lipoprotein (HDL) in the blood serum, which did not change with the low fat diet (Dashti et al., 2007; De Natale et al., 2009; Dyson et al., 2007; Haimoto et al., 2008). This is noteworthy because the nutrition intervention in diabetic patients should contribute to improvements in long term cardiovascular health.

De Natale et al (2009) had competing results due to their control diet. Their study followed ADA guidelines for recommended diet during this time period, but focused on a high carbohydrate, high fiber, low glycemic index foods diet. There was a significant decrease $(\mathrm{p}<$ 0.05) in total cholesterol, LDL, blood glucose, and triglycerides (De Natale et al., 2009). The authors concluded that a high carbohydrate and fiber diet is a useful treatment for patients with type 2 diabetes. While this study had considerably better outcomes on this diet, the study is limited due to all participants' blood glucose being well-controlled and consistent use of metformin. Additionally, the study was four weeks long and too short to show long term results or diet compliance.

Iqbal et al. (2010) did not have significant results due to a $52.8 \%$ attrition. The researchers attributed the high attrition rate to participants losing interest in the study, moving, or for unstated reasons. The study was also limited because the participants self-reported their intake, and resulted in an underestimation of macronutrient intake. The researchers disclosed that they did not verify the self-reporting of medications, and changes in health status with the participants medical records, in turn limiting the validity and reliability of the 
observations. The high attrition of participants resulted in the researchers completing a chart review on 26 of the 44 participants. Using a chart review does allow the researchers to know medications and changes to the patient's health status, but does not allow for an accurate dietary recall without the participant present.

\subsection{Recommendations}

The research studies during this time period had notable results. A low carbohydrate diet can potentially increase HDL (Dashti et al., 2007; De Natale et al., 2009; Elhayany et al., 2010; Haimoto et al., 2008). This is important because an HDL above $40 \mathrm{mg} / \mathrm{dL}$ and $50 \mathrm{mg} / \mathrm{dL}$ can decrease risk for cardiovascular disease in men and women, respectively (Mahan \& Raymond, 2016). When compared to the low fat diet, the low carbohydrate diet consistently increased the HDL. This was not found in the traditional ADA diet.

A less restrictive low carbohydrate diet (45\% of calories from carbohydrates) can lead to better compliance in diabetic patients (Haimoto et al., 2008). A 5\% reduction in carbohydrate intake in patients with diabetes can have the same results as a more restrictive diet (De Natale et al., 2009; Dyson et al., 2007; Elhayany et al., 2010; Esposito et al., 2009; Haimoto et al., 2008). A strength of the studies is the authors' use of a Registered Dietitian or nutritionist for nutrition education and dietary assessment, and importantly suggests nutrition intervention is best supported with the use of a Registered Dietitian as part of the healthcare team (De Natale et al., 2009; Dyson et al., 2007; Esposito et al., 2009; Haimoto et al., 2008). The ADA recommends medical nutrition therapy be completed on patients with diabetes.

A diet high in unsaturated fats can improve the cardiovascular profile in diabetic patients (Elhayany et al., 2010; Esposito et al., 2009). This calls attention to the combination of a low carbohydrate diet and the Mediterranean meal plan had compounded effects on cardiovascular and diabetic biomarkers. Esposito et al. (2009) point out the monounsaturated fatty acids improve the cell's uptake of insulin, and suggests a Mediterranean diet can have positive outcomes on diabetic biomarkers. A more intentional application of a low carbohydrate, high fat diet that includes avocados, nuts, seeds, olive oil and other plant-based oils that remain liquid at room temperature. This is a staple in the Mediterranean diet, along with high fruit and vegetable intake.

Overall, from 2007 to 2010 the research showed positive results in favor of low-carbohydrate diet. The low-carbohydrate diet, when compared to a low fat diet, showed improvement in HgbA1c, blood glucose, and HDL in a short period of time. In 2008 the ADA first introduced a low carbohydrate diet as a nutrition intervention (American Diabetes Association, 2008; Elhayany et al., 2010). As research continues the studies become more robust in their intervention, but continue to be limited due to reproducibility and applicability.

\section{2011-2014}

During the years of 2011-2014, the ADA defined a low-carbohydrate diet as less than 130 grams of carbohydrates per day, and a diet option for people with type 2 diabetes (American Diabetes Association, 2011). The studies completed from 2007-2010 were ground breaking in bringing to light that low-carbohydrate is a viable dietary intervention for this condition 
(Dashti et al., 2007; Dyson et al., 2007; Elhayany et al., 2010; Esposito et al., 2009; Haimoto et al., 2008). A low-carbohydrate diet is recommended for short-term use only (American Diabetes Association, 2011). This medical nutrition therapy recommendation cautioned health professionals to closely monitor the patient's lipid panel, kidney function, and onset of hypoglycemia (American Diabetes Association, 2011). Additionally, protein intake was to be monitored in patients with nephropathy. The ADA (2011) recommendations acknowledged that positive changes in serum triglyceride, HDL, HgbAlc were more pronounced in the low carbohydrate diet versus a low fat diet.

It is important to point out that the LDL is reported to increase in low-carbohydrate diets (American Diabetes Association, 2011). Increased LDL can lead to increased risk for cardiovascular disease, which is already a major concern for people with diabetes. The increased LDL related to the choice of fats diabetic patients are ingesting during these trials, further supports results from Elhayany et al (2010) and Esposito et al (2009) with patients following a Mediterranean low carbohydrate diet. A diet enriched with monounsaturated fat has been shown to increase HDL and decrease LDL (Dashti et al., 2007; Dyson et al., 2007; Elhayany et al., 2010; Esposito et al., 2009; Haimoto et al., 2008) Generally, the Mediterranean diet is higher in monounsaturated fats when compared to a more traditional western diet. The 2011 ADA recommendations continue to drive the importance of individualized nutrition therapy for patients with diabetes, and reminds professionals and patients that the Recommended Dietary Allowance (RDA) is 130 grams per day of carbohydrates (American Diabetes Association, 2011). Recommending 130 grams of carbohydrates is interesting because it is not individualized to every patient. The ADA position statement also states that there is limited research on the long-term metabolic effects on a very-low carbohydrate diet. The limited research on a very low-carbohydrate diet also supports the need to define a low-carbohydrate diet, and develop studies that are reproduceable to generate more evidence.

\subsection{Clinical Trials}

During this time period the definition of low carbohydrate diet varied between researchers, as shown in Table 2. The control diet was a low fat diet containing 20\% (Larsen, Mann, Maclean, \& Shaw, 2011), 25\% (Yamada et al., 2014), or 30\% (Fernemark et al., 2013; Guldbrand et al., 2012; Hussain et al., 2012; Mayer et al., 2014; Pearce, Clifton, \& Noakes, 2011; Rock et al., 2014; Saslow et al., 2014; Tay et al., 2014) of calories from fat, and 50\% carbohydrates (Fernemark et al., 2013; Guldbrand et al., 2012; Hussain et al., 2012; Krebs et al., 2013; Larsen et al., 2011; Mayer et al., 2014; Pearce et al., 2011; Rock et al., 2014; Saslow et al., 2014; Tay et al., 2014; Yamada et al., 2014). Saslow et al (2014) introduced a new diet category with "very low carbohydrate" diet, and his study design resulted in more powerful results. This dietary intervention was used in the preceding time period by researchers and provided less than 20g (Dashti et al., 2007) or 40g (Dyson et al., 2007) of carbohydrates. 


\section{Macrothink}

Table 2. Description of low carbohydrate interventions in studies of participants with type 2 diabetes 2011-2014

Reference

Population

Larsen et al. 108 overweight/obese participants (2011)

Pearce et al. 82 overweight/obese participants (2011) with a HbgA1c between $6.5-10 \%$, months and no significant history of heart disease, stroke within the past 3 months, or liver and renal disease with a HgbAlc less than $9 \%$, and diagnosis of type 2 diabetes. No history of liver, kidney, or gastrointestinal disease, and could not be injecting foods that could interfere with the study

\section{Study \\ Length \\ Author's description of nutrition intervention}

12

A low $\mathrm{CHO}$ was defined as low fat diet $(30 \%$ of total calories from fat) high in protein $(30 \%$ total energy)

12 weeks Two low $\mathrm{CHO}$ dietary arms defined as "high-protein

high-cholesterol diet with a $\mathrm{CHO}$, protein, fat ratio of 40:30:30\%, and 590 mg cholesterol. Animal protein diet (high-protein low-cholesterol, with $\mathrm{CHO}$, protein, fat ratio of 40:30:30, $213 \quad \mathrm{mg}$ cholesterol.

6 months Low $\mathrm{CHO}$ diet defined as $20 \%$ of calories come from $\mathrm{CHO}$ and with or without glucose-lowering medication, incretin-based therapy, or insulin

Hussain et al. 363 overweight/obese participants (2012) with 102 with type 2 diabetes and 261 without type 2 diabetes

Fernemark et al. 21 participants with type 2 (2013)
24 weeks

Low CHO ketogenic diet is defined as less than 20 grams of $\mathrm{CHO}$ per day without anti-diabetic drugs, but excluded participants using insulin or sulfonylureas 
from $\mathrm{CHO}$

Krebs et al. 14 obese participants with type 224 weeks

Low $\mathrm{CHO}$ diet defined as

diabetes and treated by either diet

fat intake greater than or required oral hypoglycemic agents or insulin $40 \%$ of calories with a reduced $\mathrm{CHO}$ intake

Mayer et al. 46 overweight/obese military (2014) veteran participants with type 2

48 weeks Low $\mathrm{CHO}$ diet is defined diabetes and did not have type 1 diabetes, unstable chronic diseases, or a disease that would interfere

as less than or equal to 20 grams $\mathrm{CHO}$ per day with unrestricted caloric intake, until goal weight was reached

Rock et al. 227 overweight/obese participants (2014) with type 2 diabetes and do not have an eating disorder, food allergies, food intolerance, or history of a bariatric surgery

Saslow et al. 34 overweight/obese participants (2014) with type 2 diabetes (HgbAlc above $6.5 \%$ ) or prediabetes (HbAlc $>6 \%$ ), and not using oral glucocorticoids or weight loss medications, vegan, taking insulin, or three oral hypoglycemic medications

Tay et al. (2014) 115 overweight/obese participants diagnosed with type 2 diabetes and/or taking antiglycemic medication and no significant chronic diseases, eating disorder, 24 weeks Low $\mathrm{CHO}$ diet is defined

12 months

Low $\mathrm{CHO}$ diet is defined as $45 \%, 25 \%, 30 \%$ of calories from $\mathrm{CHO}$, protein, fat respectively

3 months

Very Low CHO diet is defined as 20 to $50 \mathrm{~g}$ of $\mathrm{CHO}$ a day (not including fiber) as $14 \%, 28 \%, 58 \%$ of calories from $\mathrm{CHO}$, protein, fat respectively

Yamada et al. 24 Japanese participants with type (2014)

2 diabetes with an HgbAlc between $6.9-8.4 \%$

6 months

Low CHO diet is defined as less than 130 grams but greater than 70 grams total CHO per day.

Saslow et al. (2014) incorporated behavioral components to improve diet compliance on the very low carbohydrate diet. The participants received 13, two-hour classes that educated on the diet assigned, and skills supporting behavior change. The study followed the ADA 


\section{MInstitute Macrothink $_{\text {Int }}$}

recommendation of a lifestyle intervention by education on sleep, exercise, mindfulness, and realistic goal setting (American Diabetes Association, 2011; Saslow et al., 2014). The participants were instructed to change their diet by the fourth class. The change was accomplished by the researchers encouraging the participants to decrease their carbohydrate intake to less than 20-50 grams per day in 7 to 10 days (Saslow et al., 2014). The use of lifestyle interventions, behavioral components, and goal setting to increase dietary compliance out shadowed the weakness of short duration and possible reporting bias from participants.

Other study designs began to incorporate lifestyle interventions which led to a low attrition rate and statistically significant outcomes. Tay et al (2014), Pearce et al (2011), Yamada et al (2014), and Hussain et al (2012) provided meal plans, diet educations, or personalized nutrition recommendations from a Registered Dietitian. The use of Registered Dietitians increased compliance among participants and decreased attrition (Hussain et al., 2012; Pearce et al., 2011; Tay et al., 2014; Yamada et al., 2014).

Rock et al (2014) was the only study that provided all food for participants, but there were no measures to verify the food was consumed. The more rigorous study design used by Rock et al (2014) illustrates patients with diabetes require more than an interaction with their primary provider. The use of the allied health team in providing education can improve compliance, diet maintenance, and overall improvement in blood glucose control and cardiac risk factors.

Larsen et al (2011) was the only study that did not find statistically significant results between the control and intervention groups. The control and intervention group had statistically significant reduction in $\mathrm{HgbA1c}(p=0.05)$. The study results could be due to study design and participant compliance with diet. Nineteen participants did not follow the recommended diet, five had missing data, and all participants self-reported their diet.

Overall the study designs during this time period incorporated behavioral interventions and attempted to control the diet through increased diet education and mindfulness. The incorporation of behavioral and lifestyle intervention showed positive cardiac and diabetic outcomes among participants.

\subsection{Results}

All studies noted during 2011-2014 observed a reduction in HgbA1c in the control and intervention diet. The statistically significant $(p<0.05)$ reductions occurred in the low carbohydrate interventions (Guldbrand et al., 2012; Hussain et al., 2012; Krebs et al., 2013; Mayer et al., 2014; Rock et al., 2014; Tay et al., 2014; Yamada et al., 2014). Tay (2014) discovered the reduction in HgbAlc was only significant in participants with baseline HgbAlc greater than $7.8 \%(p=0.02)$. Participants with a HgbAlc less than or equal to $7.8 \%$ did not see a significant decrease $(p=0.77)$. The role of HgbA1c was not observed or noted in other studies during 2011-2014, but it may be useful to do further research and find a comparison between $\mathrm{HgBA} 1 \mathrm{c}$ and the response to a low-carbohydrate diet.

Fasting glucose was not recorded as an outcome by most studies during this time period (Guldbrand et al., 2012; Krebs et al., 2013; Larsen et al., 2011; Mayer et al., 2014; Saslow et 
al., 2014; Tay et al., 2014; Yamada et al., 2014). Rock et al (2014) led a study that compared 227 overweigh/obese patients with diabetes. The intervention group was provided food and exercise coaching. The control group was provided usual care of two weight loss consults and monthly meetings. The intervention group observed a greater reduction of fasting blood glucose versus the control group ( $p=0.023$ ).

Krebs et al. (2013) completed a study with 14 participants following a low-carbohydrate diet, with fat intake greater than $40 \%$. The researchers observed a statistically significant reduction in blood glucose in 12 weeks ( $\mathrm{p}=0.049$ ). The change in recommendations that $\mathrm{HgBA} 1 \mathrm{c}$ as a better indicator of glucose control does not discredit the need to track the blood glucose. In these studies (Krebs et al., 2013; Rock et al., 2014), changes in blood glucose showed statistical significance, regardless of the short duration.

Similar to 2007-2010, a low carbohydrate diet increases HDL blood serum levels (Guldbrand et al., 2014; Hussain et al., 2012; Krebs et al., 2013; Larsen et al., 2011; Pearce et al., 2011; Tay et al., 2014). Saslow et al (2014) observed a decrease in the HDL over 3 months in the low carbohydrate group, and Yamada et al (2014) did not observe any significant improvements in the HDL. In the Saslow et al (2014) study, the decrease in HDL could be related to reporting bias from participants and not controlling the dietary intake. The decrease in HDL was not discussed in the paper, but may be an outlier due to the majority of the studies showing an increase in HDL (Guldbrand et al., 2014; Hussain et al., 2012; Krebs et al., 2013; Larsen et al., 2011; Pearce et al., 2011; Rock et al., 2014; Tay et al., 2014).

The 2011 ADA recommendations reported LDL increased in a low carbohydrate diet, and warned against high intake. Results from the Krebs et al (2013) and Rock et al (2014) studies support the claim of an increase in LDL. Studies during this time period did not find significant increases in LDL, nor did changes differ from the control group (Hussain et al., 2012; Saslow et al., 2014). A low-carbohydrate diet can have neutral results on LDL, and depending on the diet, genetics, and activity level can cause an increase.

Most notably, the study by Pearce et al (2011) with a low carbohydrate intervention group including consuming eggs increased daily cholesterol ingested to $590 \mathrm{mg}$. Consumption at the level reported in the study is above current recommendations of less than $300 \mathrm{mg}$ of cholesterol daily (Mahan \& Raymond, 2016). The outcome of the study revealed a reduction of total cholesterol in all subjects, with HDL increasing in the low carbohydrate arm. Pearce (2011) reports that it is known that exogenous cholesterol does not have an effect on insulin-resistant patients, and it should not be surprising the same results were found in patients with type 2 diabetes.

\subsection{Recommendations}

During this time period study design improved to control food, exercise, and caloric intake (Hussain et al., 2012; Krebs et al., 2013; Pearce et al., 2011; Rock et al., 2014; Saslow et al., 2014; Tay et al., 2014; Yamada et al., 2014). Additionally, strength in the research design increased in these studies when behavioral practices, exercise, and medical nutrition therapy was integrated into the study. Pearce et al (2011) reported individualized nutrition plans for 
each participant improved compliance. Compliance of the nutrition plan is one of the key components of a lifestyle intervention to treat patients with type 2 diabetes.

For practitioners who treat patients with type 2 diabetes, it can be challenging to ensure the patients adhere to recommended dietary changes. The implementation of exercise classes (Tay et al., 2014; Yamada et al., 2014), mindfulness (Yamada et al., 2014), group classes (Guldbrand et al., 2012), and individualized nutrition plans (Fernemark et al., 2013; Hussain et al., 2012; Krebs et al., 2013; Pearce et al., 2011; Rock et al., 2014) are tools that can be used to increase dietary compliance while improving diabetic and cardiovascular biomarkers, which were highlighted during the 2011-2014 time period.

\section{2015-2019}

Starting in 2015 to 2019 the body of research has advanced significantly, and the ADA recommendations reflect the changes. The need for more research is correlated to the increase of diabetes incidence within the US. Presently two out of every five Americans will develop type 2 diabetes over their lifetime, and prevalence is increasing in children, teens, and young adults (American Diabetes Association, 2020). The 2015 ADA recommendations vary greatly from previous recommendations by focusing on patient-centered care. The recommendations do not advocate for any diet specifically, but encourage early education for self-management (American Diabetes Association, 2015). The emphasis is on the need for a lifestyle intervention through nutrition, exercise, pharmacology, and behavioral therapy (American Diabetes Association, 2015; American Diabetes Association, 2020).

HgbAlc shows blood sugar over a longer time period versus fasting blood glucose, and provide better perspective of the patient's glucose control. HgbA1c will also be an important measure to incorporate into diabetes clinical trials for the future. Due to the increased incidence of diabetes in people under the age of 18 years, the ADA defined HgbA1c for adults only. The changes to patient-centered care and HgbAlc are observed in the trials completed in the 2015-2019 time period. The therapeutic interventions are more innovative to increase dietary compliance and behavioral change, which results in positive diabetic and cardiovascular biomarkers.

The ADA outlines four goals for medical nutrition therapy for patients with diabetes, displayed in Table 3 (American Diabetes Association, 2015; American Diabetes Association, 2020). The goals highlight the importance of individualized nutrition interventions, and also de-emphasis focus on a specific macronutrient group.

Table 3. Goals of Medical Nutrition Therapy for Adults with Diabetes

1. To promote and support healthful eating patterns, emphasizing a variety of nutrient-dense foods in appropriate portion sizes, in order to improve overall health

2. To address individual nutrition needs based on personal and cultural preferences, health literacy and numeracy, access to healthful food choices, willingness and 
ability to make behavioral changes, and barriers to change

3. To maintain the pleasure of eating by providing positive messages about food choices while limiting food choices only when indicated by scientific evidence

4. To provide the individual with diabetes with practical tools for day-to-day meal planning rather than focusing on individual macronutrients, micronutrients, or single foods

The new goals instead focus on portion control and finding pleasure in eating. Previously, some patients with type two diabetes have felt very restricted to their food choices once diagnosed. The patient's needs are important to value with using a team approach for treatment (American Diabetes Association, 2015; American Diabetes Association, 2020). The philosophy change is unique from the previous ADA recommendations reviewed.

\subsection{Clinical Trials}

Beginning in 2015, clinical trials became more robust in intervention to increase participant compliance and reproducibility of the study designs. The researchers focused not only on the low-carbohydrate intervention, but using urine samples to ensure participants were in ketosis (Goday et al., 2016; Romano et al., 2019). Table 4 defines the low carbohydrate interventions for this time period.

Table 4. Description of low carbohydrate interventions in studies of participants with type 2 diabetes 2015-2019

\begin{tabular}{|c|c|c|c|}
\hline Reference & Population & Study Length & $\begin{array}{l}\text { Author's description of } \\
\text { nutrition intervention }\end{array}$ \\
\hline $\begin{array}{l}\text { Nanri et al. } \\
(2015)\end{array}$ & $\begin{array}{l}27,799 \text { men and } \\
36,875 \text { women in } \\
\text { prospective study and } \\
\text { who had no history of } \\
\text { diabetes, over } 5 \text { years } \\
\begin{array}{l}1191 \text { new cases of } \\
\text { type } 2 \text { diabetes } \\
\text { developed }\end{array}\end{array}$ & 5 years & $\begin{array}{l}\text { Low } \mathrm{CHO} \text { diet defined as high } \\
\text { total protein and fat and low } \\
\mathrm{CHO}\end{array}$ \\
\hline $\begin{array}{l}\text { Sasakabe et } \\
\text { al. }(2015)\end{array}$ & $\begin{array}{l}76 \text { participants with a } \\
\text { type } 2 \text { diabetes } \\
\text { diagnosis, and not } \\
\text { taking anti-diabetic } \\
\text { medications, severe } \\
\text { diabetic }\end{array}$ & 3 months & $\begin{array}{l}\text { Moderate low-CHO diet } \\
\text { defined as at baseline and after } \\
3 \text { months were 51:27:15 and } \\
41: 33: 18 \text { in men and 54:27:16 } \\
\text { and } 42: 37: 19 \quad \text { in } \\
\text { women, \%CHO: \%fat: \%protein }\end{array}$ \\
\hline
\end{tabular}


complications, and

lost weight within the month

Tay et al. 115 overweight/obese (2015) participants with type 2 diabetes or taking diabetic medications, and no significant health history, an eating disorder, or a smoking

Wycherley et 115 obese participants al. (2016) with type 2 diabetes who exercised 3 days a week for 60 minutes

Tay et al. 115 overweight/obese (2018) participants with type 2 diabetes and/or using diabetes medication, and without a significant health history, eating disorder, or a smoker

Brinkworth et 115 obese participants al. (2016) with type 2 diabetes with no significant health history of chronic disease, eating disorder, or smoking

Goday et al. 89 obese participant 4 months (2016) with type 2 diabetes diagnosis for less than 10 years, and not on insulin, no renal or liver disease, and without a severe eating or psychiatric disorder respectively

Low $\mathrm{CHO}$ diet defined as $14 \%$ energy as $\mathrm{CHO}, 28 \%$ as protein, $58 \%$ as fat $(<10 \%$ saturated fat)

Low $\mathrm{CHO}$ diet defined as $14 \%$ energy as $\mathrm{CHO}, 28 \%$ as protein, $58 \%$ as fat $(<10 \%$ saturated fat $)$

Low $\mathrm{CHO}$ diet defined as $14 \%$ energy as $\mathrm{CHO}, 28 \%$ as protein, $58 \%$ as fat $(<10 \%$ saturated fat)

A low $\mathrm{CHO}$ diet defined as a dietary plan aimed at providing $14 \%$ of total energy as $\mathrm{CHO}$

Very Low ketogenic diet defined as $600-800 \mathrm{kcal}$ per day with less than $50 \mathrm{CHO}$ per day 
Saslow et al. 34 overweight/obese (2017); participants with type 2 diabetes and not using insulin or more than three glucose-lowering medications.

Saslow et al. 25 overweight/obese (2017) participants with a type 2 diabetes diagnosis, internet access, and not taking metformin

Sato et al. $66 \quad$ overweight (2017) participants with type 2 diabetes and have attended a diabetes education

Bhanpuri et 262 obese participants 12 months al. (2018) with type 2 diabetes
12 months

32 weeks

6 months

Very low-carbohydrate ketogenic (LCK) diet defined as CHO intake between 20 to 50 grams per day
Very low-carbohydrate ketogenic (LCK) diet defined as $\mathrm{CHO}$ intake between 20 to 50 grams per day
Low $\mathrm{CHO}$ diet defined as 130 grams per day with liberalized calories
Francois et al. $16 \quad$ participants (2018) diagnosed to type 2 diabetes who exercise 3 days a weeks for the preceding 3 months and are not using insulin and do not have any cardiovascular, kidney or complications. 
change in oral antidiabetic drugs or in insulin half a month before the intervention, without nut allergies and difficulty chewing nuts. Does not have severe health conditions

Liu et al. 122 healthy 12 weeks (2018) participants with a BMI within normal limits and newly diagnosed with type 2 diabetes with the last 3 months, blood pressure within normal limits, and exercise, no significant health history

Myette-Côtéet 16 al. (2018) diagnosed to Type 2 diabetes not using insulin and without diagnosed cardiovascular ,kidney, or severe diabetes complications

Samkani et al. 16 (2018)

Sanada et al. 200 retrospective 36 months (2018) participants Two short term diagnosed with type 248 with intervention with diabetes by well-controlled by taking metformin, non-smokers with steady weight.

Japanese participants with type 2 diabetes, calories less than $45 \%$

Low $\mathrm{CHO}$ diet defined as $42 \%$ and $28 \%$ of calories from $\mathrm{CHO}$ and protein respectively
3 short-term 4 Consisted of two low $\mathrm{CHO}$ day intervention dietary arms defined as: periods

Low $\mathrm{CHO}$ high-fat diet with $\mathrm{CHO}$ limited to $\sim 10 \%$ of total energy and low $\mathrm{CHO}$ with 15-min post meal walks

Low $\mathrm{CHO}$ diet defined as $31 \%$ from $\mathrm{CHO}, 29 \%$ energy from protein and $40 \%$ from fat, respectively 
grams per day (2018) participants diagnosed intake of between 30-200 with type 2 diabetes, and has not had any changes to their grams per day or calories from carbohydrates/total calories of $<45 \%$

diabetic medications or insulin 2 weeks, did not have a nut allergy, no difficulties chewing or swallowing, and does not have any severe cardiovascular, kidney, or liver disease.

Romano et al. 25 participants with (2019) type 2 diabetes, being treated with diet and/or oral antidiabetics, and/or insulin, with no illness over 8 years, weight loss in the last 6 months, treatment with thiazolidinediones, glucagon-like peptide-1 agonists, steroids, or atypical antipsychotic; untreated thyroidism; renal alteration and alcohol dependency
8 weeks

Very low-calorie ketogenic diet defined as synthetic amino acid protein supplementation with less than 25 grams of $\mathrm{CHO}$ per day and lipids from EVOO

A diet containing less than $45 \%$ of calories from carbohydrates was used by various researchers (Bhanpuri et al., 2018; Francois et al., 2018; Myette-Cote et al., 2018; Nanri et al., 2015; Samkani et al., 2018; Saslow, Mason et al., 2017; Tay et al., 2015; Tay et al., 2018). The other researchers followed the 2011 ADA reported RDA for carbohydrates of 130 per day (Goday et al., 2016; Hou et al., 2018; Sanada et al., 2018). The repetitive nature of some of the dietary interventions (Saslow et al., 2017; Saslow et al., 2017; Tay et al., 2015; Tay et al., 2018; Wycherley et al., 2016) illustrated a trend to increase reproducibility of studies. 


\section{Macrothink}

During this time period researchers were recreating their study designs with more detailed interventions.

The most notable intervention included web based education and counseling (Bhanpuri et al., 2018; Saslow et al., 2017). Bhanpuri et al (2018) provided an individualized continuous care intervention online and in person. The control group received care from Registered Dietitians in accordance with the ADA guidelines (Bhanpuri et al., 2018). The intervention participants were given an option that best suited them. Then over 12 months, health coaches guided participants into nutritional ketosis, provided feedback, and online peer support (Bhanpuri et al., 2018). Nutritional ketosis is the reduction of carbohydrate intake that depletes the glycogen stores in the liver, and results in the liver breaking down fatty acids to provide energy for the body (Bhanpuri et al., 2018; Gershuni, Yan, \& Medici, 2018). The end product results in an elevated amount of beta-hydroxybutyrate in the blood stream, or ketones in the urine (Bhanpuri et al., 2018; Gershuni et al., 2018). Bhanpuri (2018) monitored the levels of beta-hydroxybutyrate in the blood stream to ensure nutritional ketosis had occurred.

Saslow et al (2017) completed a 32-week web-based intervention providing the participants with low-carbohydrate ketogenic diet or the plate method recommendations. The use of web based education and counseling can be valuable to many patients with diabetes, but this approach is limited due to ability of medical facilities to provide web based nutrition counseling consistently. The intervention may not be appropriate for people who have low income, uninsured, or who do not have regular access to the internet.

The length of clinical trials during this time period is notable. Trials varied from four days to 36 months. A four-day trial showed statistically significant results $(p<0.05)$, which is surprising considering the short intervention period (Myette-Cote et al., 2018). The participants were provided all food to increase dietary compliance, but this limits the ability to apply to all patients with diabetes and is not cost-effective (Myette-Cote et al., 2018). The study had 11 participants which is a small sample size and reduces the generalizability (Myette-Cote et al., 2018). A 36-month retrospective trial was completed with 200 Japanese participants (Sanada et al., 2018). The participants were instructed to reduce their carbohydrate intake to $20-40 \mathrm{~g}$ per meal, $70-130 \mathrm{~g}$ per day, and $10 \mathrm{~g}$ per snack. The results showed that improved cardiac and diabetic biomarkers could be maintained over a longer period of time. The length of the studies is important to note because earlier trials ranged from three to twelve months (Dyson et al., 2007; Goday et al., 2016; Guldbrand et al., 2014; Hou et al., 2018; Hussain et al., 2012; Krebs et al., 2013; Liu et al., 2018; McLaughlin et al., 2007; Myette-Cote et al., 2018; Pearce et al., 2011; Samkani et al., 2018; Saslow et al., 2017; Sato et al., 2017; Tay et al., 2014; Wang et al., 2018; Yamada et al., 2014). The limitation to long term studies is high attrition and low dietary compliance (Saslow et al., 2017; Tay et al., 2015; Tay et al., 2018). The ADA did not recommend a low-carbohydrate nutrition intervention due to limited long-term studies (American Diabetes Association, 2007; American Diabetes Association, 2008), and Sanada et al (2018) provided evidence that low-carbohydrate dietary intervention can be beneficial for patients with type 2 diabetes over a longer period of time. 


\subsection{Results}

Similar to other time periods statistically significant results were found in the low carbohydrate intervention versus the former ADA recommended intervention. HgbA1c was statistically significant $(p<0.05)$ in the low carbohydrate diet (Goday et al., 2016; Hou et al., 2018; Liu et al., 2018; Romano et al., 2019; Sanada et al., 2018; Saslow et al., 2017; Saslow et al., 2017; Sato et al., 2017; Tay et al., 2015; Wang et al., 2018). Sanada et al (2018) found HgbA1c improved within the first six months of the study, and the improvement maintained for 36 months. Sato et al (2017) observed no improvements in HgbA1c $(p<0.01)$ from the control group and is a possible outlier result related to study design, bias, or the implementation of the control diet. Liu et al (2018) showed a larger decrease in HgbA1c participants that supplemented with $\omega-3(p=0.01)$ versus the low carbohydrate group without supplementation $(p=0.03)$. The study affirms previous recommendations of considering the low carbohydrate Mediterranean diet as a dietary intervention in patients with diabetes (Elhayany et al., 2010; Esposito et al., 2009).

Comparable to the 2011-2014 timeframe, fasting blood glucose was evaluated in fewer studies as an outcome variable (Hou et al., 2018; Liu et al., 2018; Myette-Cote et al., 2018; Tay et al., 2015; Tay et al., 2018; Wang et al., 2018). The well documented reliability of the HgbAlc is a better assessment of glucose control over a long period of time (American Diabetes Association, 2015). Hou et al (2018) altered the typical low carbohydrate intervention, by requiring the participant to eat nuts in replacement of starchy food. The change in the dietary consumption caused the fasting blood glucose to be significantly lower $(p=0.001)$ three weeks into the three-month study. Myette-Côtéet et al (2018) completed a four-day study showing the fasting blood glucose can be lowered quickly with carbohydrate restriction. The results of the research have limited applicability because of the small sample group, and the short period of time the study was conducted.

Throughout the 2015-2019 time period cardiovascular biomarkers showed positive improvements in the control and intervention. Most notably HDL, when measured, increased in patients who received a low-carbohydrate diet (Bhanpuri et al., 2018; Tay et al., 2015). In addition, triglyceride reduction was observed in the low carbohydrate diet (Goday et al., 2016; Tay et al., 2015; Tay et al., 2018). Overall the cardiovascular risk is reduced with the low carbohydrate diet. This does not negate the possibility that a low-fat diet over a longer period of time may show the same results, but a low-carbohydrate diet can show cardiac and diabetic biomarker improvements faster.

\subsection{Recommendations}

The 2015 to 2019 time period provided reproduceable study designs and behavioral interventions. The addition of medical nutrition therapy goals sheds light on the importance of recommendations that focus on portion control, eating a variety of nutrient dense foods, and balanced meals that included all macronutrients (American Diabetes Association, 2015; American Diabetes Association, 2020). The studies during this time period provide food 
(Bhanpuri et al., 2018; Francois et al., 2018; Goday et al., 2016; Hou et al., 2018; Myette-Cote et al., 2018; Samkani et al., 2018) or written meal plans (Liu et al., 2018) to participants.

In daily medical practice it is not feasible or sustainable to provide food or meal plans, but the study designs emphasize the need to educate a patient on proper food choices (Bhanpuri et al., 2018; Saslow et al., 2017; Tay et al., 2015; Tay et al., 2018; Wycherley et al., 2016). The exposure to nutrition education via online or in person resulted in higher study retention and improvements in HgbA1c (Bhanpuri et al., 2018; Saslow et al., 2017; Tay et al., 2015; Tay et al., 2018; Wycherley et al., 2016). Repeated nutrition education as a part of the care provided to patients with type 2 diabetes is a necessary component to improve diabetic and cardiovascular outcomes.

\section{Discussion}

The literature review brought to light the use of a low-carbohydrate diet for individuals with type 2 diabetes. The following recommendations show the evolution of type 2 diabetes research, and provide suggestions for more in depth research regarding nutrition.

\subsection{Recommendations}

Considering the data reviewed from 2007 to present, there is evidence to support the use of a low carbohydrate diet in patients with type 2 diabetes. Most notably there was a reduction in HgbA1c, fasting blood glucose, and an increase in HDL.

According to the Standard of Care in Diabetic Patients 2015 (American Diabetes Association, 2015), weight loss is encouraged in order to improve glycemic control and reduce risk for heart disease. The low carbohydrate diet provides weight loss in most participants. This poses the question, is the improvement of diabetic and cardiovascular biomarkers related to weight loss, the nutrition intervention, or both? It is accepted within the literature that weight loss will improve symptoms of type 2 diabetes (American Diabetes Association, 2020; Dashti et al., 2007; Hamdy et al., 2018; Iqbal et al., 2010; Krebs et al., 2013; McLaughlin et al., 2007; Sanada et al., 2018; Saslow et al., 2017); however, is it necessary to differentiate the positive results?

At this time, it is not necessary to distinctly define if weight loss or the low-carbohydrate diet is the cause of improved cardiac and diabetic biomarkers. Being that obesity is the leading risk factor for type 2 diabetes, a reduction in body weight is a positive outcome to a diet intervention. Larsen et al (2011) and Sanada et al (2018) observed a diet high in protein and fat improves glycemic control, and does not solely focus on weight loss in patients with type 2 diabetes. Larsen et al (2011) supports Dashti et al (2007) argument that the low carbohydrate diet works via many possible mechanisms. The researchers proposed the weight loss following ketosis is water weight, but eventually, following a ketogenic diet can result in maintaining lean body mass while decreasing body fat (Dashti et al., 2007; Larsen et al., 2011). This in turn causes the body to use more energy and excrete it in the form of ketones in urine, sweat and feces (Dashti et al., 2007). Larsen et al (2011) expounds on this by stating that increasing protein and fat intake while lowering carbohydrate intake can produce earlier 


\section{MInstitute ${ }^{\text {Macrothink }}$}

satiety and preserve lean body mass with weight loss. This supports the idea the weight loss is not necessarily a negative outcome in a low carbohydrate diet.

Ensuring ketosis is maintained in participants was rarely accomplished throughout the literature reviewed. The focus for nutritional ketosis does not become prominent in research until after 2015. After 2015, the implementation of lifestyle intervention and more intense behavioral therapy increased the tracking of ketones. Was this a necessary step to ensure dietary compliance with a low carbohydrate intervention? Several studies provided support that tracking ketones was not necessary, but instead reducing calories from carbohydrates to $45 \%$ or less showed positive response in glycemic control and cardiovascular blood serum markers (De Natale et al., 2009; Elhayany et al., 2010; Esposito et al., 2009; Haimoto et al., 2008; Hou et al., 2018; McLaughlin et al., 2007; Pearce et al., 2011; Rock et al., 2014; Sanada et al., 2018; Saslow et al., 2014; Wang et al., 2018). This is especially important when considering dietary compliance in patients with type 2 diabetes. Dietary compliance is a challenge when making recommendations to patients with diabetes. Restricting carbohydrates to less than 50 grams per day may be difficult to sustain long term.

Conducting online distance lifestyle and nutrition interventions could be a viable nutrition intervention for patients (Bhanpuri et al., 2018; Saslow et al., 2017). Researchers have shown that dietary compliance increased, and glycemic control occurred. As technology advances and the tele-health market expands, providing distance counseling and support to patients with diabetes may be the way of the future. The challenge is determining if the platform used in the trial is reproduceable, in addition to being cost effective. Can the same model be applied throughout the United States? Will this be accessible to people who are low income, uninsured, or do not have constant access to the internet? In theory distance counseling is an innovative intervention, but reproducibility is a major limitation.

Following a plant based diet high in carbohydrates can have the same effect that a low carbohydrate diet with meat as the primary source of protein. More research needs to be conducted regarding the effect of plant-based diets on type 2 diabetes (De Natale et al., 2009). De Natale et al (2009) found that diets high in fiber and high in carbohydrates have better outcomes than a low carbohydrate diet with animal products. A high fiber diet combined with low glycemic index foods decreases the release of insulin in the body and in turn prevents spikes in blood glucose levels. In 2019, there is evidence of a plant based movement and the 2020 ADA Diabetes Recommendations added vegetarian or vegan dietary intervention in response (American Diabetes Association, 2020). The study design of De Natale et al (2009) would be ideal to reproduce in patients with poor glycemic control over a long period of time to see the effects.

\subsection{Gaps and Limitations of the Research}

The control diet has well-defined parameters, and the low carbohydrate diet had different diet variations. A more concrete definition of a low carbohydrate diet needs to be defined for future research (Hussain et al., 2012; Krebs et al., 2013; Tay et al., 2015; Wang et al., 2018; Yamada et al., 2014). Defining a low-carbohydrate diet is important because of the increased risk of hypoglycemia in patients with diabetes especially if the patient is using insulin or 
pharmacotherapy in conjunction with a low carbohydrate diet. From 2017 to present the definition varies, and the results vary. Consistently noted in the findings was a reduction in weight, HgbA1c, fasting blood glucose, and an increase of HDL. More research should be conducted to allow for a smaller reduction on carbohydrates and assess the long-term results.

Long-term studies of low carbohydrate diet are needed to evaluate the efficacy of the diet. Larsen et al (2011) pointed out that the high-carbohydrate low fat diet is regularly challenged because it can negatively affect hyperglycemia and dyslipidemia, but it is still unknown if long term carbohydrate reduction would be more beneficial. The limitation to longer-term studies is an attrition rate of higher than $30 \%$ in studies between 12 to 48 months (Elhayany et al., 2010; Haimoto et al., 2008; Iqbal et al., 2010; Sanada et al., 2018). On the other hand other studies had a low attrition rate with a 12 to 36 month time trial period, and more research is needed to evaluate the more successful study designs (Bhanpuri et al., 2018; Dashti et al., 2007; Esposito et al., 2009; Larsen et al., 2011; Rock et al., 2014; Tay et al., 2015; Wycherley et al., 2016).

\section{Conclusions}

This literature review follows the evolution of the research from 2007 to 2019, based on the status of the American Diabetes Association Standards of Care (American Diabetes Association, 2008; American Diabetes Association, 2011; American Diabetes Association, 2015; American Diabetes Association, 2020). The literature review supports a low carbohydrate diet in type two diabetic patients is more effective than following a reduced caloric, high carbohydrate intake eating pattern in the improvement of diabetic (blood glucose and HgbA1c) and cardiovascular (total cholesterol, HDL, LDL, and triglycerides) biomarkers. Type 2 diabetes is a growing concern globally. Providing patient-centered care that is individualized is important to the patient with diabetes (American Diabetes Association, 2015). A low carbohydrate or ketogenic diet is an option for diabetic patients, but it is important that the practitioner providing this recommendation consider the challenge of dietary compliance, and the varied definition of a low carbohydrate diet. The goal is to implement a lifestyle change in a person with diabetes. Presently, there are no long-term studies to support the efficacy of this intervention being utilized in adults. The review showed a consistent increase in HDL and greater reduction in HgbAlc and fasting blood glucose. A low carbohydrate dietary intervention may be an effective starting point to assist a person with diabetes to obtain blood sugar control, and then consider a more long-term intervention in the future.

\section{Acknowledgment}

The author would like to thank Dr. Haubrick, Dr. Ledoux, Mrs. Ferrell, and Mrs. Scott for their mentorship and support. She would also like to thank her mother, Kathie, for her continued support and encouragement.

\section{References}

American Diabetes Association. (2007). Standards of medical care in diabetes--2007. Diabetes Care, 31 Suppl 1(1), S12-S54. https://doi.org/10.2337/dc08-S012

American Diabetes Association. (2008). Standards of medical care in diabetes--2008. 
Diabetes Care, 31 Suppl 1(1), S12-S54. https://doi.org/10.2337/dc08-S012

American Diabetes Association. (2011). Standards of medical care in diabetes--2011. Diabetes Care, 34 Suppl 1(1), S11-S61. https://doi.org/10.2337/dc11-S011

American Diabetes Association. (2015). Standards of medical care in diabetes 2015. Diabetes Care, 38 Suppl 1(1), S1-S40. https://doi.org/10.2337/dc15-S003

American Diabetes Association. (2020). Standard of medical care in diabetes 2020. Diabetes Care, 43(1), S7-S65. https://doi.org/10.2337/dc20-S001

Bhanpuri, N. H., Hallberg, S. J., Williams, P. T., McKenzie, A. L., Ballard, K. D., Campbell, W. W., \& Volek, J. S. (2018). Cardiovascular disease risk factor responses to a type 2 diabetes care model including nutritional ketosis induced by sustained carbohydrate restriction at 1 year: An open label, non-randomized, controlled study. Cardiovascular Diabetology, 17(1), 56-8. https://doi.org/10.1186/s12933-018-0698-8

Dashti, H. M., Mathew, T. C., Khadada, M., Al-Mousawi, M., Talib, H., Asfar, S. K., \& Al-Zaid, N. S. (2007). Beneficial effects of ketogenic diet in obese diabetic subjects. Molecular and Cellular Biochemistry, 302(1-2), 249-256. https://doi.org/10.1007/s11010-007 $-9448-\mathrm{z}$

De Natale, C., Annuzzi, G., Bozzetto, L., Mazzarella, R., Costabile, G., Ciano, O., \& Rivellese, A. A. (2009). Effects of a plant-based high-carbohydrate/high-fiber diet versus high-monounsaturated fat/low-carbohydrate diet on postprandial lipids in type 2 diabetic patients. Diabetes Care, 32(12), 2168-2173.https://doi.org/10.2337/dc09-0266

Dyson, P. A., Beatty, S., \& Matthews, D. R. (2007). A low-carbohydrate diet is more effective in reducing body weight than healthy eating in both diabetic and non-diabetic subjects. Diabetic Medicine: A Journal of the British Diabetic Association, 24(12), 1430-1435. https://doi.org/10.1111/j.1464-5491.2007.02290.x

Elhayany, A., Lustman, A., Abel, R., Attal-Singer, J., \& Vinker, S. (2010). A low carbohydrate Mediterranean diet improves cardiovascular risk factors and diabetes control among overweight patients with type 2 diabetes mellitus: A 1-year prospective randomized intervention study. Diabetes, Obesity \& Metabolism, 12(3), 204-209. https://doi.org/10.1111/j.1463-1326.2009.01151.x [doi]

Esposito, K., Maiorino, M. I., Ciotola, M., Di Palo, C., Scognamiglio, P., Gicchino, M., \& Giugliano, D. (2009). Effects of a Mediterranean-style diet on the need for antihyperglycemic drug therapy in patients with newly diagnosed type 2 diabetes: A randomized trial. Annals of Internal Medicine, 151(5), 306-314. https://doi.org/10.7326/0003-4819-151-5-200909 010-00004

Fernemark, H., Jaredsson, C., Bunjaku, B., Rosenqvist, U., Nystrom, F. H., \& Guldbrand, H. (2013). A randomized cross-over trial of the postprandial effects of three different diets in patients with type 2 diabetes. PloS One, 8(11), e79324. https://doi.org/10.1371/journal.pone.0079324 


\section{Macrothink}

Francois, M. E., Myette-Cote, E., Bammert, T. D., Durrer, C., Neudorf, H., DeSouza, C. A., \& Little, J. P. (2018). Carbohydrate restriction with post meal walking effectively mitigates postprandial hyperglycemia and improves endothelial function in type 2 diabetes. American Journal of Physiology Heart and Circulatory Physiology, 314(1), H105-H113. https://doiorg/10.1152/ajpheart.00524.2017

Gershuni, V., Yan, S., \& Medici, V. (2018). Nutritional ketosis for weight management and reversal of metabolic syndrome. Current Nutrition Reports, 7(3), 97-106. https://doi.org/10.1007/s13668-018-0235-0

Goday, A., Bellido, D., Sajoux, I., Crujeiras, A. B., Burguera, B., Garcia-Luna, P. P., \& Casanueva, F. F. (2016). Short-term safety, tolerability and efficacy of a very low-calorie-ketogenic diet interventional weight loss program versus hypocaloric diet in patients with type 2 diabetes mellitus. Nutrition \& Diabetes, 6(9), e230. https://doi.org/10.1038/nutd.2016.36

Guldbrand, H., Dizdar, B., Bunjaku, B., Lindstrom, T., Bachrach-Lindstrom, M., Fredrikson, M., Nystrom, F. H. (2012). In type 2 diabetes, randomisation to advice to follow a low-carbohydrate diet transiently improves glycaemic control compared with advice to follow a low-fat diet producing a similar weight loss. Diabetologia, 55(8), 2118-2127. https://doi.rog/10.1007/s00125-012-2567-4

Haimoto, H., Iwata, M., Wakai, K., \& Umegaki, H. (2008). Long-term effects of a diet loosely restricting carbohydrates on HbAlc levels, BMI and tapering of sulfonylureas in type 2 diabetes: A 2-year follow-up study. Diabetes Research and Clinical Practice, 79(2), 350-356. https://doi.org/S0168-8227(07)00493-7

Hamdy, O., Tasabehji, M. W., Elseaidy, T., Tomah, S., Ashrafzadeh, S., \& Mottalib, A. (2018). Fat versus carbohydrate-based energy-restricted diets for weight loss in patients with type 2 diabetes. Current Diabetes Reports, 18(12), 12-4. https://doi.org/10.1007/s11892-018-1103-4

Hou, Y. Y., Ojo, O., Wang, L. L., Wang, Q., Jiang, Q., Shao, X. Y., \& Wang, X. H. (2018). A randomized controlled trial to compare the effect of peanuts and almonds on the cardio-metabolic and inflammatory parameters in patients with type 2 diabetes mellitus. Nutrients, 10(11), https://doi.org/10.3390/nu10111565.

Hussain, T. A., Mathew, T. C., Dashti, A. A., Asfar, S., Al-Zaid, N., \& Dashti, H. M. (2012). Effect of low-calorie versus low-carbohydrate ketogenic diet in type 2 diabetes. Nutrition (Burbank, Los Angeles County, Calif.), 28(10), 1016-1021. https://doi.org/10.1016/j.nut. 2012.01.016

Iqbal, N., Vetter, M. L., Moore, R. H., Chittams, J. L., Dalton-Bakes, C. V., Dowd, M., \& Wadden, T. A. (2010). Effects of a low-intensity intervention that prescribed a low-carbohydrate vs. a low-fat diet in obese, diabetic participants. Obesity (Silver Spring, Md.), 18(9), 1733-1738. https://doi.org/10.1038/oby.2009.460

Krebs, J. D., Bell, D., Hall, R., Parry-Strong, A., Docherty, P. D., Clarke, K., \& Chase, J. G. (2013). Improvements in glucose metabolism and insulin sensitivity with a low-carbohydrate 
diet in obese patients with type 2 diabetes. Journal of the American College of Nutrition, 32(1), 11-17. https://doi.org/10.1080/07315724.2013.767630

Larsen, R. N., Mann, N. J., Maclean, E., \& Shaw, J. E. (2011). The effect of high-protein, low-carbohydrate diets in the treatment of type 2 diabetes: A 12 month randomised controlled trial. Diabetologia, 54(4), 731-740. https://doi.org10.1007/s00125-010-2027-y

Liu, K., Wang, B., Zhou, R., Lang, H. D., Ran, L., Wang, J., Mi, M. T. (2018). Effect of combined use of a low-carbohydrate, high-protein diet with omega-3 polyunsaturated fatty acid supplementation on glycemic control in newly diagnosed type 2 diabetes: A randomized, double-blind, parallel-controlled trial. The American Journal of Clinical Nutrition, 108(2), 256-265. https://doi.org/10.1093/ajcn/nqy120

Mahan, L. K., \& Raymond, J. L. (2016). Krause's Food \& The Nutrition Care Process. Retrieved from http://www.vlebooks.com/vleweb/product/openreader?id=none\&isbn= 9780323340762\&uid=none

Mayer, S. B., Jeffreys, A. S., Olsen, M. K., McDuffie, J. R., Feinglos, M. N., \& Yancy, W. S., Jr. (2014). Two diets with different haemoglobin A1c and antiglycaemic medication effects despite similar weight loss in type 2 diabetes. Diabetes, Obesity \& Metabolism, 16(1), 90-93. https://doi.org/10.1111/dom.12191

McLaughlin, T., Carter, S., Lamendola, C., Abbasi, F., Schaaf, P., Basina, M., \& Reaven, G. (2007). Clinical efficacy of two hypocaloric diets that vary in overweight patients with type 2 diabetes: Comparison of moderate fat versus carbohydrate reductions. Diabetes Care, 30(7), 1877-1879. https://doi.org/10.2337/dc07-0301.

Myette-Cote, E., Durrer, C., Neudorf, H., Bammert, T. D., Botezelli, J. D., Johnson, J. D., \& Little, J. P. (2018). The effect of a short-term low-carbohydrate, high-fat diet with or without post meal walks on glycemic control and inflammation in type 2 diabetes: A randomized trial. American Journal of Physiology Regulatory, Integrative and Comparative Physiology, 315(6), R1210-R1219. https://doi.org/10.1152/ajpregu.00240.2018

Nanri, A., Mizoue, T., Kurotani, K., Goto, A., Oba, S., Noda, M., Japan Public Health Center-Based Prospective Study Group. (2015). Low-carbohydrate diet and type 2 diabetes risk in Japanese men and women: The Japan Public Health Center-based Prospective Study. PloS One, 10(2), e0118377. https://doi.org/10.1371/journal.pone.0118377

Pearce, K. L., Clifton, P. M., \& Noakes, M. (2011). Egg consumption as part of an energy-restricted high-protein diet improves blood lipid and blood glucose profiles in individuals with type 2 diabetes. The British Journal of Nutrition, 105(4), 584-592. https://doi.org/10.1017/S0007114510003983

Rock, C. L., Flatt, S. W., Pakiz, B., Taylor, K. S., Leone, A. F., Brelje, K., Sherwood, N. E. (2014). Weight loss, glycemic control, and cardiovascular disease risk factors in response to differential diet composition in a weight loss program in type 2 diabetes: A randomized controlled trial. Diabetes Care, 37(6), 1573-1580. https://doi.org/10.2337/dc13-2900 


\section{MInstitute ${ }_{\text {Mnk }}^{\text {Macrothink }}$}

Romano, L., Marchetti, M., Gualtieri, P., Di Renzo, L., Belcastro, M., De Santis, G. L.,De Lorenzo, A. (2019). Effects of a personalized VLCKD on body composition and resting energy expenditure in the reversal of diabetes to prevent complications. Nutrients, 11(7), https://doi.org/10.3390/nu11071526

Samkani, A., Skytte, M. J., Anholm, C., Astrup, A., Deacon, C. F., Holst, J. J., Haugaard, S. B. (2018). The acute effects of dietary carbohydrate reduction on postprandial responses of non-esterified fatty acids and triglycerides: A randomized trial. Lipids in Health and Disease, 17(1), 295-8. https://doi.org/10.1186/s12944-018-0953-8

Sanada, M., Kabe, C., Hata, H., Uchida, J., Inoue, G., Tsukamoto, Y., \& Yamada, S. (2018). Efficacy of a moderately low carbohydrate diet in a 36-month observational study of Japanese patients with type 2 diabetes. Nutrients, 10(5), https://doi.org/10.3390/nu10050528

Saslow, L. R., Daubenmier, J. J., Moskowitz, J. T., Kim, S., Murphy, E. J., Phinney, S. D., \& Hecht, F. M. (2017). Twelve-month outcomes of a randomized trial of a moderate-carbohydrate versus very low-carbohydrate diet in overweight adults with type 2 diabetes mellitus or prediabetes. Nutrition \& Diabetes, 7(12), 304-9. https://doi.org/10.1038 /s41387-017-0006-9

Saslow, L. R., Kim, S., Daubenmier, J. J., Moskowitz, J. T., Phinney, S. D., Goldman, V., \& Hecht, F. M. (2014). A randomized pilot trial of a moderate carbohydrate diet compared to a very low carbohydrate diet in overweight or obese individuals with type 2 diabetes mellitus or prediabetes. PloS One, 9(4), e91027. https://doi.org/10.1371/journal.pone.0091027

Saslow, L. R., Mason, A. E., Kim, S., Goldman, V., Ploutz-Snyder, R., Bayandorian, H., \& Moskowitz, J. T. (2017). An online intervention comparing a very low-carbohydrate ketogenic diet and lifestyle recommendations versus a plate method diet in overweight individuals with type 2 diabetes: A randomized controlled trial. Journal of Medical Internet Research, 19(2), e36. https://doi.org/10.2196/jmir.5806

Shamseer L, Moher D, Clarke M, Ghersi D, Liberati A, Petticrew M, Shekelle P, Stewart L, PRISMA-P Group. Preferred reporting items for systematic review and meta-analysis $\begin{array}{lll}\text { protocols } & \text { (PRISMA-P) } & 2015 .\end{array}$ http://www.prisma-statement.org/documents/PRISMA-P-checklist.pdf

Moskowitz, J. T. (2017). An online intervention comparing a very low-carbohydrate ketogenic diet and lifestyle recommendations versus a plate method diet in overweight individuals with type 2 diabetes: A randomized controlled trial. Journal of Medical Internet Research, 19(2), e36. https://doi.org/10.2196/jmir.5806

Sato, J., Kanazawa, A., Makita, S., Hatae, C., Komiya, K., Shimizu, T.,Watada, H. (2017). A randomized controlled trial of $130 \mathrm{~g} /$ day low-carbohydrate diet in type 2 diabetes with poor glycemic control. Clinical Nutrition (Edinburgh, Scotland), 36(4), 992-1000. https://doi.org/S0261-5614(16)30169-8

Tay, J., Luscombe-Marsh, N. D., Thompson, C. H., Noakes, M., Buckley, J. D., Wittert, G. A., \& Brinkworth, G. D. (2014). A very low-carbohydrate, low-saturated fat diet for type 2 


\section{Macrothink}

Journal of Food Studies

ISSN 2166-1073 2020, Vol. 9, No. 1

diabetes management: A randomized trial. Diabetes Care, 37(11), 2909-2918. https://doi.org/10.2337/dc14-0845

Tay, J., Luscombe-Marsh, N. D., Thompson, C. H., Noakes, M., Buckley, J. D., Wittert, G. A., Brinkworth, G. D. (2015). Comparison of low- and high-carbohydrate diets for type 2 diabetes management: A randomized trial. The American Journal of Clinical Nutrition, 102(4), 780-790. https://doi.org/10.3945/ajcn.115.112581

Tay, J., Thompson, C. H., Luscombe-Marsh, N. D., Wycherley, T. P., Noakes, M., Buckley, J. D., \& Brinkworth, G. D. (2018). Effects of an energy-restricted low-carbohydrate, high unsaturated fat/low saturated fat diet versus a high-carbohydrate, low-fat diet in type 2 diabetes: A 2-year randomized clinical trial. Diabetes, Obesity \& Metabolism, 20(4), 858-871. https://doi.org/10.1111/dom.13164

Wang, L. L., Wang, Q., Hong, Y., Ojo, O., Jiang, Q., Hou, Y. Y., \& Wang, X. H. (2018). The effect of low-carbohydrate diet on glycemic control in patients with type 2 diabetes mellitus. Nutrients, 10(6), 661. https://doi.org/10.3390/nu10060661

Wycherley, T. P., Thompson, C. H., Buckley, J. D., Luscombe-Marsh, N. D., Noakes, M., Wittert, G. A., \& Brinkworth, G. D. (2016). Long-term effects of weight loss with a very-low carbohydrate, low saturated fat diet on flow mediated dilatation in patients with type 2 diabetes: A randomised controlled trial. Atherosclerosis, 252, 28-31. https://doi.org/S0021 $-9150(16) 31212-6$

Yamada, Y., Uchida, J., Izumi, H., Tsukamoto, Y., Inoue, G., Watanabe, Y., \& Yamada, S. (2014). A non-calorie-restricted low-carbohydrate diet is effective as an alternative therapy for patients with type 2 diabetes. Internal Medicine (Tokyo, Japan), 53(1), 13-19. https://doi.org/DN/JST.JSTAGE/internalmedicine/53.0861

\section{Glossary}

ADA: American Diabetes Association

BMI: Body Mass Index

CHO: Carbohydrate

CPG: Casual plasma glucose

EVOO: Extra virgin olive oil

FPG: $\quad$ Fasting plasma glucose

HDL: High density lipoprotein

LDL: Low density lipoprotein

MUFA: Monounsaturated fatty acids

RD: Registered Dietitian 


\section{Macrothink

Journal of Food Studies

\section{Copyright Disclaimer}

Copyright for this article is retained by the author(s), with first publication rights granted to the journal.

This is an open-access article distributed under the terms and conditions of the Creative Commons Attribution license (http://creativecommons.org/licenses/by/3.0/). 